IOS Press

\title{
Automated quantification of DNA aneuploidy by image cytometry as an adjunct for the cytologic diagnosis of malignant effusion
}

\author{
Zhilan Meng ${ }^{\mathrm{a}}$, Jie Shi ${ }^{\mathrm{a}}$, Chenyan Zhu ${ }^{\mathrm{b}}$, Jiangang $\mathrm{Gu}^{\mathrm{a}}$ and Chen Zhou ${ }^{\mathrm{c}, *}$ \\ ${ }^{a}$ Department of Pathology, Peking Union Medical College Hospital, Chinese Academy of Medical Sciences \\ and Peking Union Medical College, Beijing, China \\ ${ }^{\mathrm{b}}$ Motic Pathology Diagnostic Center, Xiamen, China \\ ${ }^{\mathrm{c}}$ Department of Pathology, University of British Columbia, Vancouver, BC, Canada
}

Received: June 26, 2013; Accepted: October 14, 2013

\begin{abstract}
DNA aneuploidy is a cancer biomarker, which may have a potential diagnostic value in body effusion specimen. DNA aneuploidy is determined by measuring the DNA content of tested cells and comparing them with diploid cells (2c). In order to assess the value of automated DNA image cytometry (DNA-ICM) in the cytologic diagnosis of effusion, we measured DNA ploidy using an automated DNA-ICM analysis system in 126 consecutive effusion specimens and followed the cases for histologic diagnosis. Half of each effusion specimen was used to prepare cytologic smears for conventional cytologic diagnosis, while the other half was used to prepare a monolayer slide stained by Feulgen stain for automated ICM. By using Youden index, we found that 4 cells exceeding $2.5 \mathrm{c}$ is the optimal cut off value for aneuploidy, which has a sensitivity of $88.3 \%$ and specificity of $100 \%$ for diagnosis of malignant effusion. We also found that the DNA aneuploidy thresholds used for other types of cytologic specimens cannot be used in the diagnosis of effusion specimens. Our study demonstrated that automated DNA image cytometry is a simple, practical and cost-effective method for adjunct diagnosis of malignant effusion.
\end{abstract}

Keywords: Aneuploidy, DNA image cytometry, cytology, effusion

\section{Introduction}

Malignant effusion is the accumulation of exudates in pleural, abdominal or pericardial cavities, characterized by presence of malignant cells within the body cavities. It is commonly seen in carcinomas of lung, breast, stomach, ovary and endometrium, either as a presenting sign or part of advanced metastatic disease. It is also often the presenting symptom of malignant mesothelioma. Prompt and accurate diagnosis of malignant effusion is clinically critical, as it represents cancer in advanced stage and requires immediate treatment $[1,2]$.

*Corresponding author: Chen Zhou, Department of Pathology, University of British Columbia, Vancouver V6T 1Z3, Canada. Tel.: +6048776000; Fax: +6048776178; E-mail: czhou@bccancer.bc.ca.
Although cytologic examination of body cavity fluids is a widely used routine clinical diagnostic method for the detection of malignant cells in effusion, the sensitivity of conventional cytology is low, ranging from $30 \%$ to $60 \%[3,4]$. The accuracy of cytologic diagnosis of effusion depends on sampling, technique used for preparation of cytology slide and experience of cytopathologists. Despite the development of new techniques and improvement in immunohistochemistry, distinguishing reactive mesothelial proliferation from mesothelioma and distinguishing mesothelial cells from metastatic adenocarcinoma remain diagnostic problems. While many experimental or molecular techniques may have potential values in assisting the cytologic diagnosis of effusions and increasing its diagnostic accuracy, only a few of them could be applied in the "real world" or in routine clinical practice in cytology laboratory, when costs of equipment 
and reagents, training of staff and efficacy of diagnosis are considered [3].

Automated quantitative image analysis of DNA aneuploidy of cells has recently been shown to be a simple, practical and effective method for application of the biomarker in cytologic diagnosis [5-7]. DNA aneuploidy is a hall marker of cancer, resulting from genetic and chromosome instability of cancer cells, a common event in caner carcinogenesis [8]. DNA aneuploidy has been reported as a prognostic and predictive biomarker of cancer. It was shown to be a predictive marker for progression of dysplasia in cervical cancer [9], transformation from dysplasia to malignancy in esophageal adenocarcinoma [10], and requirement of aggressive treatment in breast [11], ovarian [12] and endometrial cancers [13] as it is associated with adverse prognosis in those and other cancers [14]. Several recent studies have shown the value of automated quantitative analysis of DNA aneuploidy by image cytometry (ICM) in the cytologic diagnosis and screening of cervical, esophageal and oral cancer [5-7, 15], among others.

In order to assess the value of DNA aneuploidy determined by automated DNA-ICM in cytologic diagnosis of effusion, we conducted a study of 126 cases of effusion by conventional cytology and automated DNA-ICM, and estimated the optimal diagnostic threshold values of automated DNA-ICM for effusion cytology using follow up histology result as the gold standard.

\section{Materials and methods}

\subsection{Patients and cytology specimens}

This study was reviewed and approved by the Ethical Committee, Peking Union Medical Hospital, Beijing. Between December 2010 and October 2011, a total of 126 consecutive patients who had body cavity fluid were included in the study. Among the patients, $75 \mathrm{had}$ pleural effusion, 47 had ascites and 4 had pericardial effusion. 49 patients were male and 77 patients were female. The age distribution of patients ranged from 25 to 82 years, with a median of 59 years old. All of the effusion specimens were received fresh without mixing with anticoagulant, and were processed immediately. The specimens were centrifuged for five minutes at $2500 \mathrm{rpm} / \mathrm{min}$. The supernatant was discarded. Half of the sediment was used to prepare for cytology smears. This was done by transferring the sediment onto glass slide and spreading the sediment evenly between two slides by gently pulling them apart. The cytology smears were stained with hematoxylin and eosin $(\mathrm{H} / \mathrm{E})$. The other half of the sediment was placed into an alcohol based fixative. After vortex and centrifugation, an aliquot of 25 microliters of the cell pellet was placed on to a glass slide to make a monolayer slide for DNA staining.

\subsection{Cytology diagnosis}

The cytology smears stained with H/E staining were used for cytologic diagnosis. We divided the cytologic diagnosis into 4 classes: 1 . Negative: effusions without presence of abnormal or malignant cells; 2. Atypical: presence of abnormal but no definite malignant cells; 3 . Suspicious: presence of only sparse severely abnormal cells or equivocal malignant cells; 4 . Positive: effusions containing unequivocal malignant cells. Cytologic diagnosis was made without knowledge of DNA aneuploidy result. Accuracy of cytologic diagnosis was compared with histologic diagnosis in tissue biopsy and surgical resection specimen from clinical follow-up assessment of all cases for about half a year.

\subsection{Automated DNA ICM}

DNA staining was carried out using the Feulgen method under temperature-controlled conditions according to the protocol of a DNA quantification kit (Motic, Xiamen, China). In brief, the slides were fixed in $10 \%$ buffered formalin, placed in $5 \mathrm{~N} \mathrm{HCl}$ at $27^{\circ}$ for one hour for acid hydrolysis, then stained in Feulgen solution for another hour. They were then rinsed thoroughly with water for 15 minutes. Measurement of DNA ploidy of cells on the slide was carried out by an automated DNA image cytometry system (MotiClassify system, Motic Medical Diagnostic Service Inc., Xiamen, China). The system consisted of a computer controlled motorized microscope and high-resolution digital camera for image scanning and capturing, and software for controlling of image scanning, processing and analysis. The software used for the automatic classification of cells, measurement of DNA ploidy and analysis of aneuploidy was derived from the British Columbia Cancer Agency, Vancouver, Canada [16]. For each slide, 1000 cell nuclei or all of the cell nuclei on the slide were scanned and 
nuclear images were collected into a nuclear DNA image gallery. The nuclei were automatically classified into normal epithelial/mesothelial nuclei, lymphocytes, neutrophils, and abnormal nuclei, according to a set of nuclear parameters, including nuclear morphology, nuclear texture features and nuclear DNA content. The specimens were considered satisfactory if at least 200 nuclei of mesothelial or epithelial cells were collected. Nuclei of rat hepatocytes were used as external control and for calibration of equipment to reach a coefficient of variation of less than $5 \%$. The guidelines in the consensus report on DNA measurements were followed $[17,18]$. The DNA content of each cell nucleus was measured and DNA ploidy was recorded as a "c" value. A DNA ploidy of $2 \mathrm{c}$ indicates a normal diploid cell, and 4c indicates a tetraploid cell.

The DNA measurement was carried out by a member of study team who had no knowledge of the results of convention cytologic diagnosis of the cases.

\subsection{Estimate of optimal DNA ploidy thresholds for diagnosis of effusion}

An aneuploid specimen was defined as a cytology specimen containing more than a predetermined number of cells whose DNA content exceeded a threshold value. We compared the DNA aneuploidy results of each case with its histologic diagnosis in follow-up tissue biopsy or surgical resection specimen, using the latter as the gold standard. We calculated the sensitivities and specificities of DNA aneuploidy for cytologic diagnosis of effusion by varying the threshold for the 2 parameters: the critical DNA content threshold for classifying a single nucleus as aneuploid and the number of aneuploid cells in a sample. Cellular DNA content thresholds ranging from $2.5 \mathrm{c}$ to $9 \mathrm{c}$ and aneuploid cell count thresholds ranging from 1 to 20 cells were used for the calculation. The optimal thresholds of DNA aneuploidy for the cytologic diagnosis of effusion were identified by analysis of the sensitivity and specificity of the combination of the 2 parameters.

\subsection{Comparison with different aneuploidy criteria for cytologic diagnosis of cancer}

Previous studies have reported cut off values of 3 cells with DNA ploidy index above $5 \mathrm{c}$ for cervical and esophageal cancer detection [5, 16], 3 cells with DNA ploidy index above 4.6c for oral cancer detection [7] as well as 1 cell exceeding 9c for tumor detection by DNA ploidy [19]. Sensitivity, specificity, PPV and NPV of DNA aneuploidy for diagnosis of effusion were also calculated using the previously described criteria of DNA image cytometry and compared with the optimal threshold values we identified in this study.

\subsection{Statistical analysis}

Sensitivity (Se), specificity (Sp), positive predictive value (PPV), and negative predictive value (NPV) were calculated for different diagnostic criteria using DNA image cytometry. Although the areas under the ROC curve (AUC) is the most commonly used index of diagnostic accuracy, the Youden Index (YI) is also frequently used [20]. The YI is defined as $\mathrm{YI}=$ Sensitivity + Specificity -1 and ranges between 0 and 1. Complete separation of the distributions of the marker values for the diseased and healthy populations results in $\mathrm{YI}=1$, whereas complete overlap gives $\mathrm{YI}=0$. In this study, we used the YI as it estimates diagnostic accuracy similar to AUC but also has other attractive features not present in the AUC. YI provides a criterion for choosing the "optimal" threshold value, the threshold value for which $\mathrm{Se}(\mathrm{c})+\mathrm{Sp}(\mathrm{c})-1$ is maximized. YI also requires no additional information such as prevalence rate and decision error cost.

\section{Results}

\subsection{Cytologic diagnosis and follow up histologic diagnosis}

Out of 126 cases, 26 showed no presence of abnormal or tumor cells and were diagnosed by conventional cytology as "negative", which on follow up showed 1 small cell carcinoma and 1 lung adenocarcinoma histologically. 1 case showed an excessive amount of lymphocytes and was diagnosed by conventional cytology as "suspicious", which on follow up lymph node biopsy was diagnosed histologically as lymphadenitis. 99 cases were diagnosed by conventional cytology as "positive" (Table 1). Among the cytology positive cases, 57 were adenocarcinomas either from ovary or lungs, confirmed by subsequent tissue biopsy or surgical resection; 2 cases were small cell carcinomas confirmed by lung biopsy and 3 cases were lymphomas (2 large B cell lymphoma and $1 \mathrm{~T}$ cell lymphoma) con- 
firmed by lymph node biopsy. For 3 cases, we applied immunohistochemistry using cell block. One of these was diagnosed by immunohistochemistry as malignant mesothelioma, while the other two were diagnosed as clear cell carcinomas from ovary and uterine cervix, all of them were confirmed in follow-up histologic diagnosis. The remaining 34 cytology positive cases could not be clearly classified or traced to their origins. The sensitivity and specificity of the conventional cytology with the assistance of immunohistochemistry are $98 \%$ and $96 \%$, respectively, if the suspicious cytology diagnosis is counted as positive.

\subsection{Diagnosis of malignant effusion by DNA aneuploidy}

Out of the 126 cases submitted for the DNA ICM analysis, 9 cases were inadequate for the analysis because of insufficient cell numbers. Only 117 cases were included in this study. The cytologic diagnosis for the 117 cases were as follows: 21 negative cases; 96 positive cases which included 58 adenocarcinoma, 3 small cell carcinoma, 2 lymphoma, 1 clear cell carcinoma, 1 mesothelioma and 31 of malignancy of

Table 1

Cytologic diagnosis of effusion

\begin{tabular}{llc}
\hline & Cytologic diagnosis & Numbers of cases \\
\hline Positive & Adenocarcinoma & 57 \\
& Small cell carcinoma & 2 \\
& Mesothelioma & 1 \\
& Lymphoma & 3 \\
& Clear cell carcinoma & 2 \\
& Unclassifiable & 34 \\
Suspicious & & 1 \\
Atypical & & 0 \\
Negative & & 26 \\
Total & & 126 \\
\hline
\end{tabular}

\section{Sensitivity}
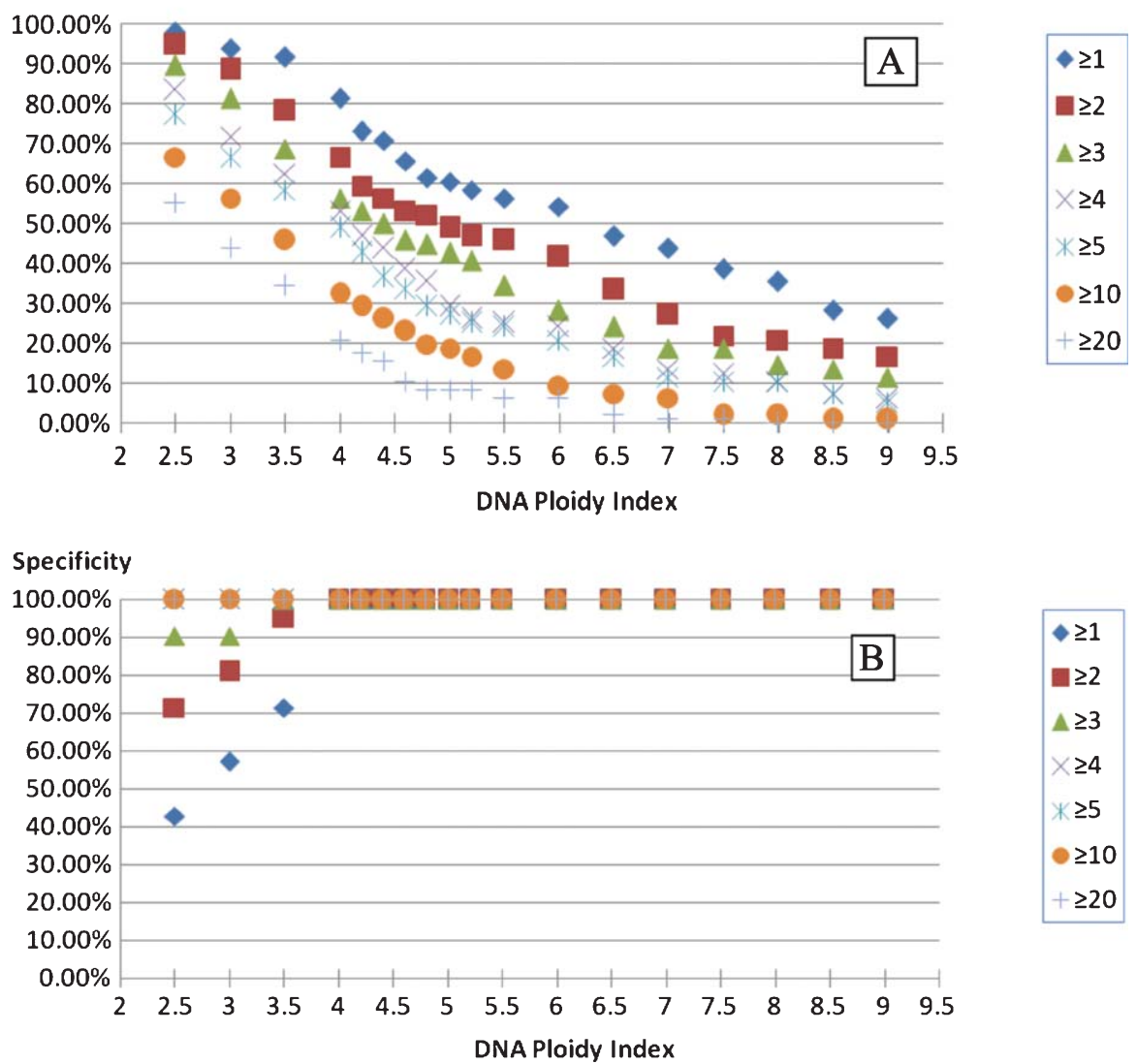

Fig. 1. Sensitivity (A) and specificity (B) of diagnosis of effusion by DNA ploidy expressed as cut off values of DNA ploidy index (ranging from $2.5 \mathrm{c}$ to $9 \mathrm{c}$ ) and minimum number of cells (ranging from 1 to 20 cells). 


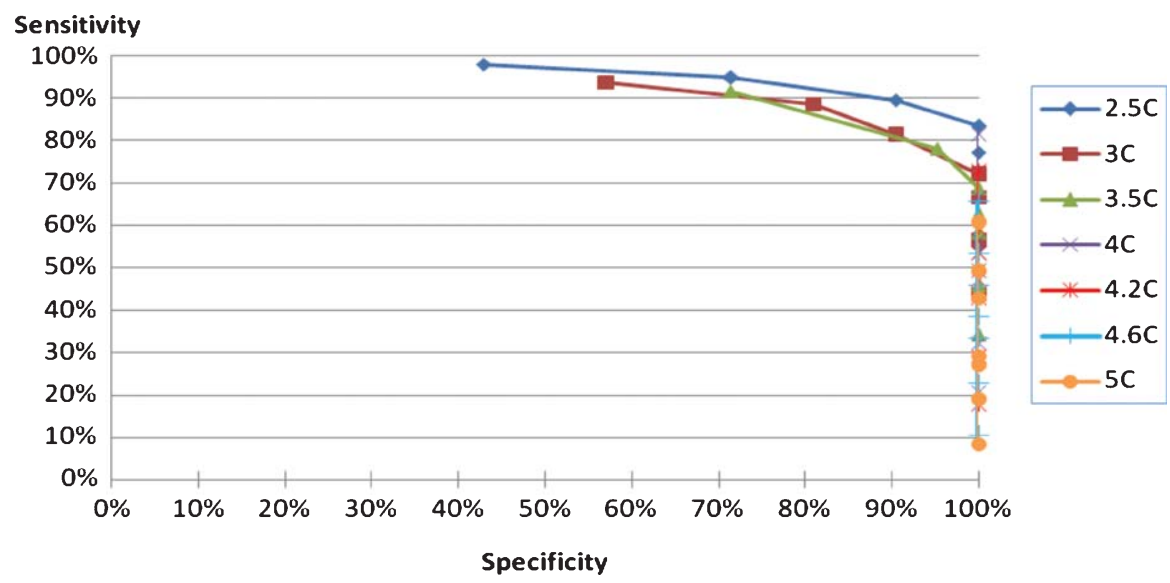

Fig. 2. The sensitivity and specificity of diagnosis of effusion by DNA ploidy, expressed as DNA ploidy index and numbers of cells exceeding such index. DNA ploidy index ranges $\mathrm{g}$ from $2.5 \mathrm{c}$ to $5 \mathrm{c}$ and cell numbers ranges from 1 to 4 . Based on Youden Index, the point (4 cells and $2.5 \mathrm{c}$ ) is at the right corner of the coordinate, which means this is the most optimal cut-off point for DNA ploidy diagnosis of effusions in this study.

unknown primary. Table 2 and Fig. 1 show the sensitivity and the specificity of DNA aneuploidy using various thresholds based on combinations of the 2 parameters of ploidy.

\subsection{Optimization of DNA ploidy parameters for diagnosis of malignant effusion}

Figure 2 showed that when the number of cells with DNA ploidy $2.5 \mathrm{c}$ was more than 4 cells, the YI was maximized, with a corresponding sensitivity and specificity of $83.3 \%$ and $100 \%$, respectively. Therefore, the optimal cut off threshold value of DNA image cytometry in effusion diagnosis was 4 cells with DNA ploidy exceeding $2.5 \mathrm{c}$.

\subsection{Comparison with different aneuploidy criteria for cytologic diagnosis of malignant effusion}

Table 3 lists the cut off threshold values for different kinds of cancers detected by DNA aneuploidy using image cytometry in comparison to the optimal threshold value of DNA aneuploidy for diagnosis of effusion we identified in this study. The result indicates that threshold value of 4 cells exceeding $2.5 \mathrm{c}$ has much better sensitivity and specificity as compared to the criteria used for other types of cytology specimens or cancer detection.

\section{Discussion}

For many years, cytologic examination of effusion cell either by conventional cytology smear or by monolayer preparation has been the principal method for diagnosis of malignant effusion [21]. However, the ability of visual detection of cancer cells in effusion samples is heavily dependent on the diagnostic skill and experience of cytopathologists. Low sensitivity in effusion cytology by conventional cytology in some situations may be due to inexperienced cytopathologists and lack of an adjunct tool for cytologic diagnosis of effusion [22, 23].

As a proven cancer biomarker, DNA aneuploidy has a potential to become an adjunct for routine cytologic diagnosis of effusion. DNA aneuploidy is assessed by measurement of DNA ploidy in cells and by comparing them with diploid cells. Both flow cytometry and image cytometry (ICM) can be used to measure DNA ploidy for the cytometric diagnosis of cancer. Previous studies using DNA ploidy for diagnosis of effusion showed that ICM was more sensitive than flow cytometry [24, 25]. Motherby et al. [25] measured DNA ploidy in 200 effusion cytology specimens and found that the sensitivity and specificity were $75 \%$ and $100 \%$ by ICM but were $32.1 \%$ and $100 \%$ by flow cytometry. By using ICM for quantification of DNA aneuploidy, several other studies showed that DNA aneuploidy was more sensitive than conventional cytology for detection of malignant cells in effusion specimen [19, 26, 27]. El-Habashi et al. [27] reported that sensitivity, 
Table 2

Sensitivity, specificity and Youden Index of cytologic diagnosis of effusion by DNA ploidy using different DNA ploidy parameters obtained from automated DNA-image cytometry

\begin{tabular}{|c|c|c|c|c|c|c|}
\hline DNA ploidy $(\mathrm{C})$ & Number of cells & $\mathrm{TP}$ & $\mathrm{TN}$ & $\mathrm{Se}$ & $\mathrm{Sp}$ & Youden index \\
\hline 2.5 & $\geq 1$ & 91 & 9 & $97.85 \%$ & $37.50 \%$ & $35.35 \%$ \\
\hline 2.5 & $\geq 2$ & 88 & 15 & $94.62 \%$ & $62.50 \%$ & $57.12 \%$ \\
\hline 2.5 & $\geq 3$ & 83 & 19 & $89.25 \%$ & $79.17 \%$ & $68.41 \%$ \\
\hline 2.5 & $\geq 4$ & 77 & 21 & $82.80 \%$ & $87.50 \%$ & $70.30 \%$ \\
\hline 2.5 & $\geq 5$ & 73 & 23 & $78.49 \%$ & $95.83 \%$ & $74.33 \%$ \\
\hline 2.5 & $\geq 10$ & 63 & 23 & $67.74 \%$ & $95.83 \%$ & $63.58 \%$ \\
\hline 2.5 & $\geq 20$ & 53 & 24 & $56.99 \%$ & $100.00 \%$ & $56.99 \%$ \\
\hline 3 & $\geq 1$ & 89 & 14 & $95.70 \%$ & $58.33 \%$ & $54.03 \%$ \\
\hline 3 & $\geq 2$ & 82 & 17 & $88.17 \%$ & $70.83 \%$ & $59.01 \%$ \\
\hline 3 & $\geq 3$ & 75 & 19 & $80.65 \%$ & $79.17 \%$ & $59.81 \%$ \\
\hline 3 & $\geq 4$ & 67 & 22 & $72.04 \%$ & $91.67 \%$ & $63.71 \%$ \\
\hline 3 & $\geq 5$ & 63 & 23 & $67.74 \%$ & $95.83 \%$ & $63.58 \%$ \\
\hline 3 & $\geq 10$ & 54 & 24 & $58.06 \%$ & $100.00 \%$ & $58.06 \%$ \\
\hline 3 & $\geq 20$ & 42 & 24 & $45.16 \%$ & $100.00 \%$ & $45.16 \%$ \\
\hline 3.5 & $\geq 1$ & 85 & 15 & $91.40 \%$ & $62.50 \%$ & $53.90 \%$ \\
\hline 3.5 & $\geq 2$ & 73 & 21 & $78.49 \%$ & $87.50 \%$ & $65.99 \%$ \\
\hline 3.5 & $\geq 3$ & 64 & 22 & $68.82 \%$ & $91.67 \%$ & $60.48 \%$ \\
\hline 3.5 & $\geq 4$ & 59 & 23 & $63.44 \%$ & $95.83 \%$ & $59.27 \%$ \\
\hline 3.5 & $\geq 5$ & 55 & 23 & $59.14 \%$ & $95.83 \%$ & $54.97 \%$ \\
\hline 3.5 & $\geq 10$ & 44 & 24 & $47.31 \%$ & $100.00 \%$ & $47.31 \%$ \\
\hline 3.5 & $\geq 20$ & 33 & 24 & $35.48 \%$ & $100.00 \%$ & $35.48 \%$ \\
\hline 4 & $\geq 1$ & 75 & 21 & $80.65 \%$ & $87.50 \%$ & $68.15 \%$ \\
\hline 4 & $\geq 2$ & 63 & 23 & $67.74 \%$ & $95.83 \%$ & $63.58 \%$ \\
\hline 4 & $\geq 3$ & 54 & 24 & $58.06 \%$ & $100.00 \%$ & $58.06 \%$ \\
\hline 4 & $\geq 4$ & 51 & 24 & $54.84 \%$ & $100.00 \%$ & $54.84 \%$ \\
\hline 4 & $\geq 5$ & 47 & 24 & $50.54 \%$ & $100.00 \%$ & $50.54 \%$ \\
\hline 4 & $\geq 10$ & 31 & 24 & $33.33 \%$ & $100.00 \%$ & $33.33 \%$ \\
\hline 4 & $\geq 20$ & 20 & 24 & $21.51 \%$ & $100.00 \%$ & $21.51 \%$ \\
\hline 4.2 & $\geq 1$ & 68 & 22 & $73.12 \%$ & $91.67 \%$ & $64.78 \%$ \\
\hline 4.2 & $\geq 2$ & 57 & 24 & $61.29 \%$ & $100.00 \%$ & $61.29 \%$ \\
\hline 4.2 & $\geq 3$ & 51 & 24 & $54.84 \%$ & $100.00 \%$ & $54.84 \%$ \\
\hline 4.2 & $\geq 4$ & 45 & 24 & $48.39 \%$ & $100.00 \%$ & $48.39 \%$ \\
\hline 4.2 & $\geq 5$ & 41 & 24 & $44.09 \%$ & $100.00 \%$ & $44.09 \%$ \\
\hline 4.2 & $\geq 10$ & 28 & 24 & $30.11 \%$ & $100.00 \%$ & $30.11 \%$ \\
\hline 4.2 & $\geq 20$ & 17 & 24 & $18.28 \%$ & $100.00 \%$ & $18.28 \%$ \\
\hline 4.4 & $\geq 1$ & 66 & 22 & $70.97 \%$ & $91.67 \%$ & $62.63 \%$ \\
\hline 4.4 & $\geq 2$ & 54 & 24 & $58.06 \%$ & $100.00 \%$ & $58.06 \%$ \\
\hline 4.4 & $\geq 3$ & 48 & 24 & $51.61 \%$ & $100.00 \%$ & $51.61 \%$ \\
\hline 4.4 & $\geq 4$ & 42 & 24 & $45.16 \%$ & $100.00 \%$ & $45.16 \%$ \\
\hline 4.4 & $\geq 5$ & 35 & 24 & $37.63 \%$ & $100.00 \%$ & $37.63 \%$ \\
\hline 4.4 & $\geq 10$ & 25 & 24 & $26.88 \%$ & $100.00 \%$ & $26.88 \%$ \\
\hline 4.4 & $\geq 20$ & 15 & 24 & $16.13 \%$ & $100.00 \%$ & $16.13 \%$ \\
\hline 4.6 & $\geq 1$ & 62 & 23 & $66.67 \%$ & $95.83 \%$ & $62.50 \%$ \\
\hline 4.6 & $\geq 2$ & 51 & 24 & $54.84 \%$ & $100.00 \%$ & $54.84 \%$ \\
\hline 4.6 & $\geq 3$ & 44 & 24 & $47.31 \%$ & $100.00 \%$ & $47.31 \%$ \\
\hline 4.6 & $\geq 4$ & 37 & 24 & $39.78 \%$ & $100.00 \%$ & $39.78 \%$ \\
\hline 4.6 & $\geq 5$ & 32 & 24 & $34.41 \%$ & $100.00 \%$ & $34.41 \%$ \\
\hline 4.6 & $\geq 10$ & 22 & 24 & $23.66 \%$ & $100.00 \%$ & $23.66 \%$ \\
\hline 4.6 & $\geq 20$ & 10 & 24 & $10.75 \%$ & $100.00 \%$ & $10.75 \%$ \\
\hline 4.8 & $\geq 1$ & 58 & 23 & $62.37 \%$ & $95.83 \%$ & $58.20 \%$ \\
\hline 4.8 & $\geq 2$ & 50 & 24 & $53.76 \%$ & $100.00 \%$ & $53.76 \%$ \\
\hline 4.8 & $\geq 3$ & 43 & 24 & $46.24 \%$ & $100.00 \%$ & $46.24 \%$ \\
\hline 4.8 & $\geq 4$ & 34 & 24 & $36.56 \%$ & $100.00 \%$ & $36.56 \%$ \\
\hline
\end{tabular}


Table 2

(Continued)

\begin{tabular}{|c|c|c|c|c|c|c|}
\hline DNA ploidy (C) & Number of cells & $\mathrm{TP}$ & $\mathrm{TN}$ & $\mathrm{Se}$ & $\mathrm{Sp}$ & Youden index \\
\hline 4.8 & $\geq 5$ & 28 & 24 & $30.11 \%$ & $100.00 \%$ & $30.11 \%$ \\
\hline 4.8 & $\geq 10$ & 19 & 24 & $20.43 \%$ & $100.00 \%$ & $20.43 \%$ \\
\hline 4.8 & $\geq 20$ & 8 & 24 & $8.60 \%$ & $100.00 \%$ & $8.60 \%$ \\
\hline 5 & $\geq 1$ & 57 & 23 & $61.29 \%$ & $95.83 \%$ & $57.12 \%$ \\
\hline 5 & $\geq 2$ & 47 & 24 & $50.54 \%$ & $100.00 \%$ & $50.54 \%$ \\
\hline 5 & $\geq 3$ & 41 & 24 & $44.09 \%$ & $100.00 \%$ & $44.09 \%$ \\
\hline 5 & $\geq 4$ & 28 & 24 & $30.11 \%$ & $100.00 \%$ & $30.11 \%$ \\
\hline 5 & $\geq 5$ & 26 & 24 & $27.96 \%$ & $100.00 \%$ & $27.96 \%$ \\
\hline 5 & $\geq 10$ & 18 & 24 & $19.35 \%$ & $100.00 \%$ & $19.35 \%$ \\
\hline 5 & $\geq 20$ & 8 & 24 & $8.60 \%$ & $100.00 \%$ & $8.60 \%$ \\
\hline
\end{tabular}

Table 3

Diagnostic accuracy using DNA ploidy diagnostic threshold values of different kinds of cytologic specimens

\begin{tabular}{lcrrrr}
\hline DNA ploidy (c) Cell numbers Sensitivity Specificity & PPV & NPV \\
\hline 2.5 & 4 & $83.3 \%$ & $100.0 \%$ & $100.0 \%$ & $53.5 \%$ \\
4.6 & 5 & $33.3 \%$ & $100.0 \%$ & $100.0 \%$ & $24.7 \%$ \\
5 & 3 & $42.7 \%$ & $100.0 \%$ & $100.0 \%$ & $27.6 \%$ \\
9 & 1 & $26.0 \%$ & $100.0 \%$ & $100.0 \%$ & $25.0 \%$ \\
\hline
\end{tabular}

specificity, PPV and NPV of DNA ploidy and conventional cytology were $85 \%$ vs. $65 \%, 94 \%$ vs. $100 \%$, $96 \%$ vs. $100 \%$ and $80 \%$ vs. $62 \%$ in 87 consecutive effusion specimens. Motherby et al. [19] reported that DNA aneuploidy defined by the presence of $9 \mathrm{c}$ cells or variation of the first DNA stemline by $\geq 10 \%$ has $100 \%$ PPV for adenocarcinoma and mesothelioma and $100 \% \mathrm{NPV}$ for adenocarcinoma and 88\% NPV for mesothelioma. Even in equivocal cases, sensitivity and specificity of DNA aneuploidy were $55.9 \%$ and $94.1 \%$, respectively [26]. However, almost all of the previous studies used manual measurement of DNA ploidy [19, 24, 26, 27]. Manual ICM is time consuming, subject to sampling error, and has to be done by an experienced cytopathologist. Thus, the cost for manual DNA ICM was higher than that of immunohistochemistry. Due to these limitations, manual DNA ICM has not been widely applied in routine diagnosis of effusion specimens.

Our study is the first to apply fully automated ICM to measure DNA ploidy in effusion cytology specimens and to determine its value in the diagnosis of effusion. Automated DNA ICM technology has been recently proven in some studies to increase the sensitivity of cytologic detection of dysplastic and malignant cells in several types of cancers $[5,6,28]$. Because it uses automation and digital slide scanning technol- ogy, ICM is simple and easy to use, requiring minimal training and experience. It is also objective and fast, taking less than 5 to 10 minutes from automatic scanning of cells on the whole slide to generating a DNA aneuploidy report. The cost for the procedure is also reduced significantly as it does not require experienced cytopathologists to consume excessive amount of time to perform manual selection and measurement of DNA ploidy of individual cells. In our study, we applied automated DNA ICM to the quantification of DNA aneuploidy in 126 consecutive effusion cytology specimens and followed up the cases for confirmation of histologic diagnosis. From those 117 cases that contained the required numbers of cells in the effusion specimens, we calculated the optimal diagnostic threshold value by the Youden Index method, which was the first time this method was used to determine the optimal threshold value of aneuploidy for cytologic diagnosis. Our result showed that the optimal threshold was 4 cells with DNA ploidy exceeding $2.5 \mathrm{c}$. Using this cut off value, the sensitivity and specificity for diagnosis of effusion were $83.3 \%$ and $100 \%$, respectively. The sensitivity and specificity of our study are similar to the $75-88 \%$ sensitivity and high specificity reported by previous studies using manual DNA ICM for effusion diagnosis $[19,25,27]$. Our study showed that automated DNA ICM could be applied to effusion cytology diagnosis with similar diagnostic accuracy as compared to manual ICM.

In our study, we found that DNA threshold value for effusion diagnosis is $2.5 \mathrm{c}$. This is somewhat similar to the DNA Index 1.15 (2.3c) reported by Osterheld et al. [29] in a study using semi-automatic DNA ICM on Feulgen stained cytology slides in 42 effusion specimens. However, our threshold value is quite different from $9 \mathrm{c}$ or $\geq 10 \%$ variation in first DNA stem line, 
the cut off value reported by Motherly et al. [19] in a study of 310 cases of effusion specimens using manual ICM method. The difference of DNA threshold values between our study and previous studies done by manual methods may be due to differences in cytology slide preparation, DNA staining method used and manual selection of cell for measurement of DNA. Automated DNA ICM on a monolayer cytology slide stained by Feulgen staining described in our study may offer a more reproducible method for aneuploidy quantification as compared to manual DNA ICM on cytology smear of an air dried cytology slide.

In our study, we found that the cut off value of $5 \mathrm{c}$ for aneuploidy determined by automated DNA ICM used for cervical and esophageal cancer detection [5, $6,16]$ and 4.6c use for oral cancer detection should not be used as threshold values for effusion cytology diagnosis, as it is not as robotic as the $2.5 \mathrm{c}$ we identified. This is due to the fact that malignant cells in cervical, esophageal and oral cancer are squamous cell carcinoma while malignant cells in effusion are largely adenocarcinoma.

Immunohistochemistry analysis using cellblock is a powerful adjunct tool in effusion cytology. A panel of antibodies including Ber Ep4, MOC 31, calretinin, CK5/6, WT-1 and others has been shown to have high sensitivity and specificity for differential diagnosis between reactive mesothelial cells and metastatic adenocarcinoma in effusion specimen [22, 23]. In our study, we applied immunohistochemistry in 3 difficult cases for differential diagnosis. The high sensitivity and specificity in our cytology diagnosis is the result of a combination of conventional cytology and immunohistochemistry. Indeed, a few earlier studies have found that a combination of Ber EP4 antibody immunostain with DNA aneuploidy analysis significantly increased the sensitivity and specificity of cytologic diagnosis of effusion [30, 31]. However, immunohistochemistry may not be available in some situations, when there is not enough cells present in cytology sample, or when cytology sample is inadequately or poorly fixed before being received by the pathology laboratory, and in most of the small hospitals in China and developing countries, as they do not have automated immunostainers or access to a full list of the antibody panel needed for quality immunohistochemistry. The cost of a full panel of immunohistochemistry for effusion diagnosis is now higher than that of automated ICM. In addition, immunohistochemistry is not useful for distinction of reactive mesothelial cells from malignant mesothelioma. In those situations, DNA aneuploidy analysis could provide a practical and effective adjunct for cytologic diagnosis of effusion.

In conclusion, this study demonstrated that automated DNA-ICM is a simple, easy to use and practical method for adjunct cytologic diagnosis of effusion. Optimal threshold value for effusion cytology specimen is different from those used for other cytology specimens. A cut off value of 4 cells with ploidy exceeding $2.5 \mathrm{c}$ is the optimal threshold value for the cytologic diagnosis of malignant effusion.

\section{References}

[1] E.E. McGrath and P.B. Anderson, Diagnosis of pleural effusion: A systematic approach, Am J Crit Care, 20(2) (2011), 119-127; quiz 128.

[2] J.T. Kaifi, et al., Multidisciplinary management of malignant pleural effusion, J Surg Oncol 105(7) (2012), 731-738.

[3] K.B. Sriram, et al., Diagnostic molecular biomarkers for malignant pleural effusions, Future Oncol 7(6) (2011), 737-752.

[4] A. Segal, et al., A diagnosis of malignant pleural mesothelioma can be made by effusion cytology: Results of a 20 year audit, Pathology 45(1) (2013), 44-48.

[5] L. Zhao, et al., Population-based study of DNA image cytometry as screening method for esophageal cancer, World $J$ Gastroenterol 18(4) (2012), 375-382.

[6] H. Tong, et al., DNA ploidy cytometry testing for cervical cancer screening in China (DNACIC Trial): A prospective randomized, controlled trial, Clin Cancer Res 15(20) (2009), 6438-6445.

[7] G. Bradley, et al., Abnormal DNA content in oral epithelial dysplasia is associated with increased risk of progression to carcinoma, Br J Cancer 103(9) (2010), 1432-1442.

[8] D.J. Gordon, B. Resio and D. Pellman, Causes and consequences of aneuploidy in cancer, Nat Rev Genet 13(3) (2012), 189-203.

[9] D. Demirel, N. Akyurek and I. Ramzy, Diagnostic and prognostic significance of image cytometric DNA ploidy measurement in cytological samples of cervical squamous intraepithelial lesions, Cytopathology 24(2) (2013), 105-112.

[10] J.M. Dunn, et al., Image cytometry accurately detects DNA ploidy abnormalities and predicts late relapse to high-grade dysplasia and adenocarcinoma in Barrett's oesophagus following photodynamic therapy, Br J Cancer 102(11) (2010), 1608-1617.

[11] A.E. Pinto, et al., DNA ploidy is an independent predictor of survival in breast invasive ductal carcinoma: A long-term multivariate analysis of 393 patients. Ann Surg Oncol 20(5) (2012), 1530-1537.

[12] M.S. Flezar, et al., Flow and image cytometric DNA ploidy: Including $5 \mathrm{c}$ exceeding cells, of serous borderline malignant ovarian tumors, Correlation with clinicopathologic characteristics. Anal Quant Cytol Histol 25(3) (2003), 139-145.

[13] M. Pradhan, et al., Prognostic importance of DNA ploidy and DNA index in stage I and II endometrioid adenocarcinoma of the endometrium, Ann Oncol 23(5) (2012), 1178-1184. 
[14] V. D'Urso, et al., Cytometry and DNA ploidy: Clinical uses and molecular perspective in gastric and lung cancer, $J$ Cell Physiol 222(3) (2010), 532-539.

[15] M.J. McCullough and C.S. Farah, The assessment of the DNA content of oral cytology via virtual microscopy for the early detection of epithelial dysplasia and neoplasia in oral mucosal lesions, Respiratory Care Clinics of North America 45(9) (2009), e114-e115.

[16] M. Guillaud, et al., DNA ploidy compared with human papilloma virus testing (Hybrid Capture II) and conventional cervical cytology as a primary screening test for cervical high-grade lesions and cancer in 1555 patients with biopsy confirmation, Cancer 107(2) (2006), 309-318.

[17] A. Bocking, F. Giroud and A. Reith, Consensus report of the ESACP task force on standardization of diagnostic DNA image cytometry, European Society for Analytical Cellular Pathology. Anal Cell Pathol 8(1) (1995), 67-74.

[18] G. Haroske, et al., Fourth updated ESACP consensus report on diagnostic DNA image cytometry, Anal Cell Pathol 23(2) (2001), 89-95.

[19] H. Motherby, et al., Static DNA cytometry as a diagnostic aid in effusion cytology: I, DNA aneuploidy for identification and differentiation of primary and secondary tumors of the serous membranes. Anal Quant Cytol Histol 20(3) (1998), 153-161.

[20] R. Fluss, D. Faraggi and B. Reiser, Estimation of the Youden Index and its associated cutoff point, Biom J 47(4) (2005), 458-472.

[21] T.C. Pereira, et al., The diagnosis of malignancy in effusion cytology: A pattern recognition approach, Adv Anat Pathol 13(4) (2006), 174-184

[22] S.R. Chowdhuri, et al., Adenocarcinoma cells in effusion cytology as a diagnostic pitfall with potential impact on clinical management: A case report with brief review of immunomarkers, Diagn Cytopathol (2012), Nov 16 [Epub ahead of print].

[23] O. Lin, Challenges in the interpretation of peritoneal cytologic specimens, Arch Pathol Lab Med 133(5) (2009), 739-742.

[24] L. Kapusta, et al., The role of DNA flow and image cytometry in the evaluation of body cavity fluids, Anal Quant Cytol Histol 13(3) (1991), 187-192.

[25] H. Motherby, et al., Diagnostic DNA-flow- vs, -imagecytometry in effusion cytology. Anal Cell Pathol 24(1) (2002), $5-15$.

[26] H. Motherby, et al., Static DNA cytometry as a diagnostic aid in effusion cytology: II, DNA aneuploidy for identification of neoplastic cells in equivocal effusions. Anal Quant Cytol Histol 20(3) (1998), 162-168.

[27] A.H. el-Habashi, et al., DNA ploidy and proliferating cell nuclear antigen image analysis of peritoneal and pleural effusions, A possible diagnostic role. Acta Cytol 41(3) (1997), 636-648.

[28] A. Torres-Rendon, et al., DNA ploidy analysis by image cytometry helps to identify oral epithelial dysplasias with a high risk of malignant progression, Oral oncology 45(6) (2009), 468-473.

[29] M.C. Osterheld, C. Liette and M. Anca, Image cytometry: An aid for cytological diagnosis of pleural effusions, Diagn Cytopathol 32(3) (2005), 173-176.

[30] K.W. Matter-Walstra and R. Kraft, Atypical cells in effusions: Diagnostic value of cell image analysis combined with immunocytochemistry, Diagn Cytopathol 15(4) (1996), 263-269.

[31] H. Motherby, et al., Immunocytochemistry and DNA-image cytometry in diagnostic effusion cytology. II. Diagnostic accuracy in equivocal smears. Anal Cell Pathol, 19(2) (1999), 59-66. 


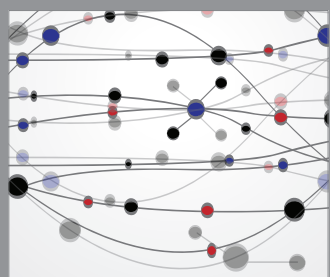

The Scientific World Journal
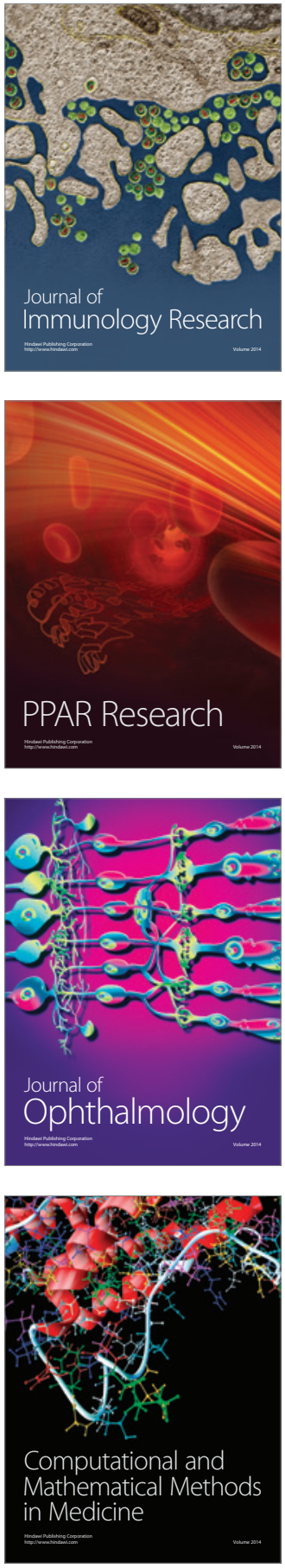

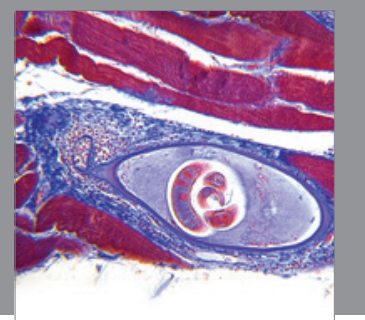

Gastroenterology

Research and Practice
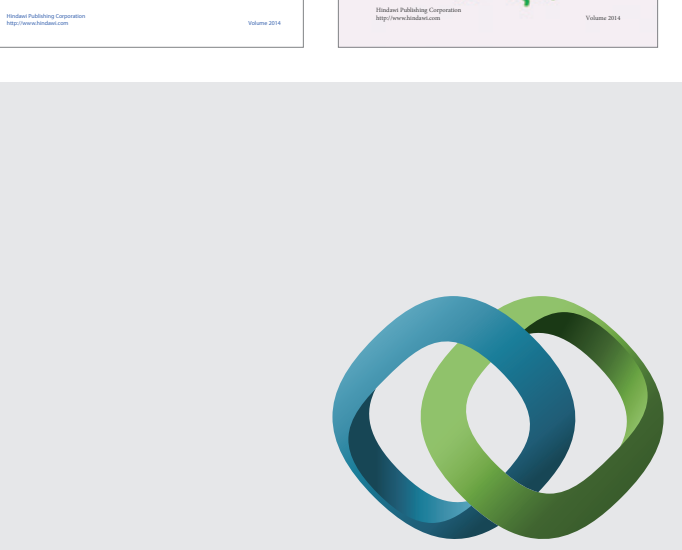

\section{Hindawi}

Submit your manuscripts at

http://www.hindawi.com
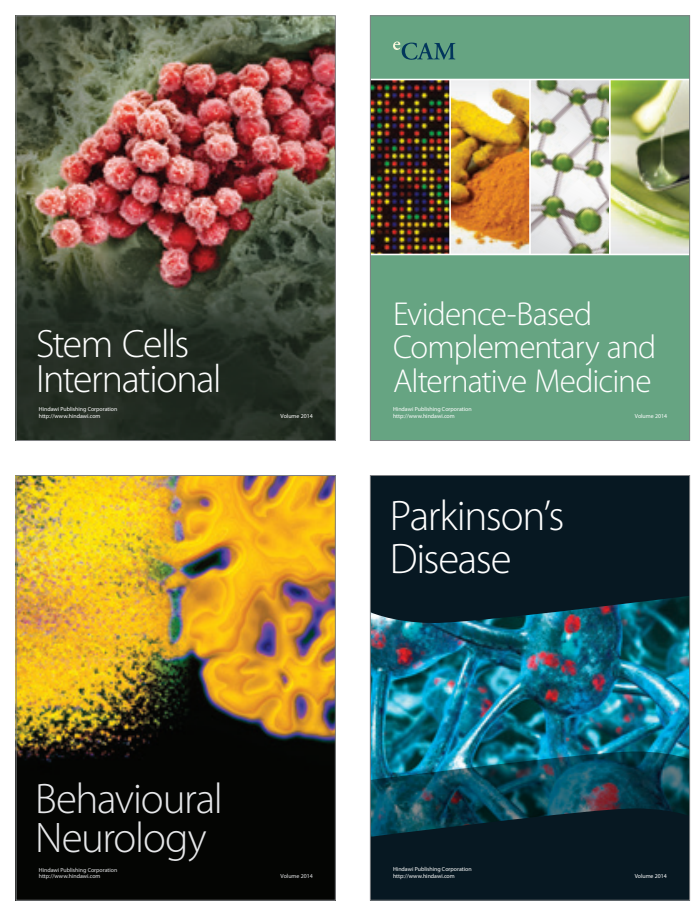

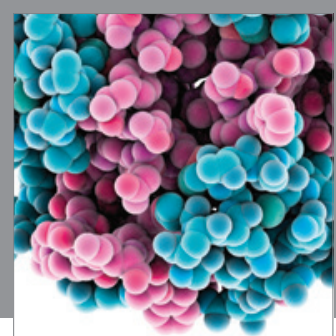

Journal of
Diabetes Research

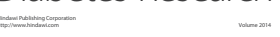

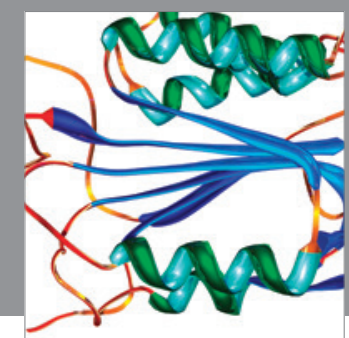

Disease Markers
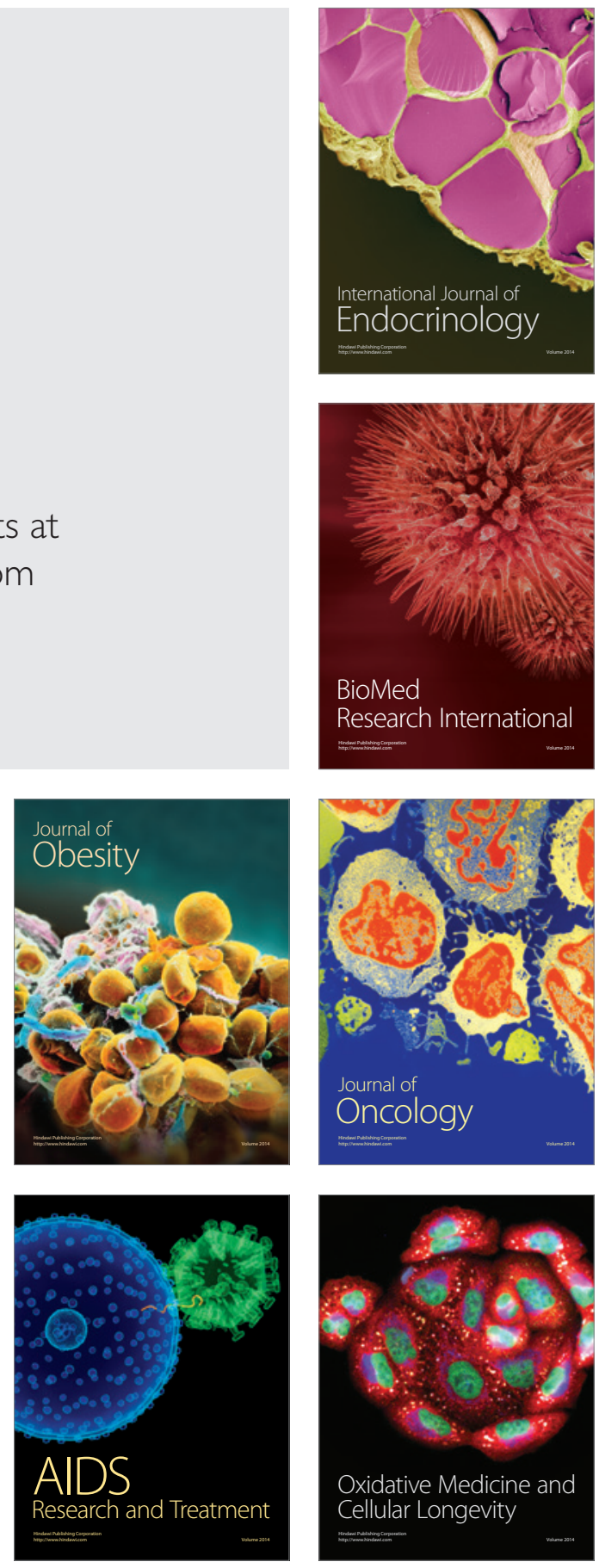